\title{
Case Study on Pyogenic Meningitis
}

Arthanareeswaran $\mathrm{S}^{1}$, Anandkumar $\mathrm{S}^{2^{*}}$, Nandhini P. $\mathrm{M}^{2}$, Akila $\mathrm{V}^{2}$, Devi Shri G. $\mathrm{S}^{2}$

${ }^{1}$ Consultant Physician, Vivekanandha Medical Care Hospital, Tiruchengode - Namakkal - Trichy Rd, Elaiyampalayam, Tamil Nadu 637205, India

${ }^{2}$ Swamy Vivekanandha College of Pharmacy, Elayampalayam-637205, Tiruchengode, Namakkal, Tamil Nadu, India

DOI: $10.36347 /$ sjmcr.2021.v09i01.026

| Received: 16.01.2021 | Accepted: 27.01.2021 | Published: 30.01.2021

*Corresponding author: Anandkumar S

Abstract

Pyogenic Meningitis is a Life-Threatening Disease which affects meninges by causing inflammation. There are 3 bacteria's which mainly causes Bacterial Meningitis, that is Haemophilus influenza, Streptococcus pneumoniae and Neisseria meningitidis. Here we have discussed about a 20 years old female patient who was diagnosed with Pyogenic Meningitis.

Keywords: Pyogenic Meningitis, Neisseria Meningiditis, Vancomycin.

Copyright $\odot 2021$ The Author(s): This is an open-access article distributed under the terms of the Creative Commons Attribution 4.0 International License (CC BY-NC 4.0) which permits unrestricted use, distribution, and reproduction in any medium for non-commercial use provided the original author and source are credited.

\section{INTRODUCTION}

Pyogenic Meningitis, also referred as Bacterial Meningitis. It is a life-threatening CNS infectious disease affecting the meninges, with elevated mortality and disability rates. It is an inflammation of meninges affecting Pia, Arachnoid and subarachnoid space. Mainly three bacteria such as Haemophilus influenza, Streptococcus pneumoniae and Neisseria meningitidis which affects for majority of cases [1,4]. Meningitis is common in children and young adults, Immunocompromised individuals, travellers, technologists, host Genetic Factors and Anatomical Defects of the Natural Barriers of the Central Nervous System and Smoking. Common Signs and Symptoms include Head and neck stiffness, Photophobia, Fever, Children - irritability, drowsiness and rash, Hyperpyrexia, Headache and Vomitting. Commonly Prescribed drugs for bacterial meningitis include Glycopeptide antibiotics like Vancomycin, Sulfonamides, Fluoroquinolones and Tetracyclines. Here, we had discussed about Patient with Pyogenic Meningitis [2, 3].

\section{Case Description}

A 20yrs old female patient got admitted in the ICU with the history of fever, headache, vomiting and altered sensorium for past 4 days. On physical examination the patient was conscious, oriented, afebrile and no Pallor. Her Vital Signs shows BP (100/60 mmHg), PR (86 bts/min), RR (20 brs/min) and Temperature (99.2 F). Her Laboratory Examination was found to have Slightly Decreased RBC, HCT, Blood urea, Serum creatinine, Serum Sodium, Serum
Potassium, Serum Chloride and WBC was increased. Her MRI of Brain (plain and contrast) showed Subtle leptomeningeal enhancement seen along tentorial surface and parieto temporal regions lightly meningitis - needs medical correlation, MR Angiography of circle of Willis shows patent major branches. Her culture report shows Gram stain - Neisseria meningitidis. The organism had disseminated to the blood. Finally, she was diagnosed with Pyogenic Meningitis.

She was treated with the following medications and it was given on all days of admission. Inj.Vancomycin $1 \mathrm{~g}$ IV BD, it is used to treat serious infections and her symptoms had improved when compared with the before days. Inj. Ceftriaxone $2 \mathrm{~g}$ IV $\mathrm{BD}$, it is used for infections caused by bacteria and her infection was reduced when compared with the first day. Tab.Pantoprazole 40mg PO OD, it reduces the amount of acid present in stomach.

Tab.Paracetamol + Domperidone PO SOS, was given on first two days of admission and it is used to treat fever and vomiting, it was stopped on $3^{\text {rd }}$ day and was changed into Paracetamol 650mg and Ondansetron $4 \mathrm{mg}$ and it was used in relieving pain caused by headache and vomiting and her symptoms was reduced when compared to before days of admission. The patient was discharged after 10 days. Her Discharge Medication Shows Inj. Vancomycin $1 \mathrm{~g}$ IV BD was given for 2 days after discharge. Tab.Pantoprazole 40mg PO OD, it reduces the amount of acid present in stomach, it was given for 7 days after discharge. Cap. Nurokind Gold, it was given after 
discharge for 7 days and it is used for the treatment of vitamin and mineral deficiencies.

\section{DISCUSSION}

Anouk M. Oordt-Speets et al., says the most frequently causing pathogens in meningitis is Neisseria Meningitidis across geographical regions [7]. AparnaYerramilli et al., in their study the ADA (Adenosine Deaminase) is found mainly in $\mathrm{T}$ lymphocytes and it is an indicator for cell mediated immunity [8]. Olaf Hoffman says, initial clinical features for Pyogenic Meningitis is non-specific which may include fever, headache, malaise, vomiting, and signs of meningitis irritation [2]. SepidehElyasi et al., shows that most commonly prescribed empirical antibiotic in treatment of Pyogenic Meningitis was Ceftriaxone combination with cefoperazone followed by Vancomycin. Treating with antibiotic increases debris including DNA. Primary treatment with antibiotic is peremptory it should not be delayed because of diagnostic parameters. Primary antibiotic treatment should be based on Empirical therapy. Prior to this Blood Culture report should be obtained to confirm the type of Specimen. Vancomycin is considered as an essential part of empirical therapy. From recent guidelines Recommend higher dose of vancomycin to achieve trough levels $15-20 \mathrm{mg} \backslash \mathrm{L}$ for severe infections. On the other side, vancomycin nephrotoxicity levels should also be monitored [9].

R Mani et al., shows Neisseria Meningitidis showed resistance to Penicillin. Other gram-negative bacilli demonstrated a high percentage of resistance to many popular drugs tested; ampicillin (79 per cent), amikacin (26.3 per cent), gentamicin (21 per cent), ciprofloxacin (47.4 per cent), axacin (58 per cent), cefotaxime (52.6 per cent), ceftazidime (63.2 per cent) and ceftriaxone (52.6 percent) [1].

Maria Karanika et al., their findings suggest that the three most frequent causative pathogens are Neisseria meningitidis $(63.0 \%)$, Streptococcus pneumoniae (14.0\%), Haemophilusinfluenzae (18.9\%) and 'other pathogens' (4.1\%) [10].

\section{Conclusion}

As Neisseria Meningitidis is emerging as the highest causative organism among other affecting bacilli, good Clinical result for Neisseria Meningitis is based on early diagnosis and initiation of effective treatment with antibiotics.

\section{REFERENCE}

1. Mani R, Pradhan S, Nagarathna S, Wasiulla R, Chandramuki A. Bacteriological profile of community Acquired acute Bacterial Meningitis. Indian Journal of Medical Microbiology. 2007; 25(2):108-14.

2. Hoffman O, Weber RJ. Pathophysiology and treatment of bacterial meningitis. Therapeutic Advances in Neurological Disorders. 2009; 2(6):401-412.

3. Bashir H El, Laundy M, Booy R. Diagnosis and treatment of bacterial meningitis. Arch Dis Child. 2003; 88:615 - 620.

4. Adriani KS, Brouwer MC, van de Beek D. Risk factors for community-acquired bacterial meningitis in adults. The Netherlands Journal of Medicine.2015; 73(2): 53-60.

5. Gump DW. Vancomycin for Treatment of Bacterial Meningitis. Reviews of Infectious Diseases. 2015; 3:289-292.

6. Furth AMV, Rood JJ, Furth RV. Roles of Proinflammatory and Anti-Inflammatory Cytokines in Pathophysiology of Bacterial Meningitis and Effect of Adjunctive Therapy. American Society for Microbiology. 1996; 64(12): 4883-4890.

7. Oordt-SpeetsAM, Bolijn $\mathrm{R}$, van Hoorn RC, Bhavsar A, Kyaw MH. Global etiology of bacterial meningitis: A systematic review and meta-analysis. Plos One. 2018; 1-16.

8. Yerramilli A, Mangapati P, Prabhakar S, Sirimulla H, Vanam S, Voora Y. A study on the clinical outcomes and management of meningitis at a tertiary care centre. Neurology India. 2017 Sep 1;65(5):1006-1012.

9. Elyasi S, Khalili H, Khavidaki S, Emadi-Koochak H. Conventional- versus high-dose vancomycin regimen in patients with acute bacterial meningitis: a randomized clinical trial. Expert Opin. Pharmacother. 2015; 16(3):297-304.

10. Karanika M, Vasilopoulou VA, Katsioulis AT, Papastergiou P, Theodoridou MN, Hadjichristodoulou CS. Diagnostic Clinical and Laboratory Findings in Response to Predetermining Bacterial Pathogen: Data from the Meningitis Registry. 2009; 4(7):1-12. 